\title{
EVIDENCIAS DE SEGMENTACIÓN Y PERSISTENCIA DE EXCLUSIÓN LABORAL EN ARGENTINA
}

\section{EVIDENCE OF SEGMENTATION AND PERSISTENT LABOR EXCLUSION IN ARGENTINA}

\section{María Eugenia Sconfienza ${ }^{1}$}

\begin{abstract}
Resumen
La población de trabajadores en Argentina comprende segmentos más y menos favorecidos. En un período en el cual una parte importante de los indicadores laborales mejoraron, amerita la reflexión de la persistencia de estructuras segmentadas. Los años recientes presentan evidencias que muestran la perseverante precarización y fragmentación del colectivo de trabajadores más pobres.
\end{abstract}

En este documento se describen, a partir de los datos proporcionados por la Encuesta Permanente de Hogares (EPH) que elabora periódicamente el Instituto Nacional de Estadística y Censos de Argentina (INDEC), algunos indicadores del mercado de trabajo observados entre 2004 y 2012 que estarían evidenciando esta dinámica. Período en el que se aplicaron un conjunto de medidas de protección socioeconómicas que propiciaron cierta recuperación luego de la crisis acontecida a principios del siglo.

Se observa que el colectivo de trabajadores que presenta condiciones de mayor precariedad es aquel compuesto por quienes detentan menores niveles educativos (en forma más marcada para el caso de las mujeres), se desenvuelven en establecimientos más pequeños, y poseen menores niveles de calificación (los no calificados duplicarían el nivel de informalidad respecto de los profesionales).

Doi: http://dx.doi.org/10.15359/eys.21-50.6

Fecha de recepción: 17-10-2016. Fechas de reenvíos: 21-10-2016 / 24-10-216 / 24-12-2016 / 25-10-2016 / $19-12-$ 2016 / 26-12-2016. Fecha de aceptación: 28-12-2016. Fecha de publicación: 28-12-2016.

${ }^{1}$ PhD. en Ciencias Sociales (Flacso). Licenciada en Ciencia Política. Trabaja en Centro de Estudios e Investigaciones Laborales del Consejo Nacional de Investigaciones Científicas y Técnicas (CEIL - CONICET), Argentina. Correo electrónico eugeniasconfienza@gmail.com

María Eugenia Sconfienza

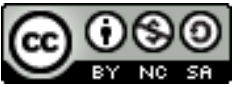

Revista Economía y Sociedad by Universidad Nacional is licensed under a CreativeCommons Reconocimiento-NoComercial- 
Palabras claves: segmentación; desempleo; exclusión.

\begin{abstract}
The population of workers in Argentina is comprised of advantaged and disadvantaged sectors. In a period in which an important part of labor indicators have improved, persistent segmentation merits reflection. Recent years show evidence of persisting precariousness and fragmentation of the poorer working class.
\end{abstract}

Based on the data from the Permanent Household Survey (EPH) periodically prepared by the National Statistics and Census Bureau in Argentina (INDEC), this paper describes some labor market indicators observed between 2004 and 2012 which evidenced this dynamic. It was in this period when a set of socio-economic protection measures were applied, which led to some recovery after the crisis at the beginning of the century.

It is observed that workers with the most precarious conditions are those with lower levels of education (particularly women), work in smaller businesses, and are less qualified (those not qualified doubled the level of informality with respect to professionals).

Keywords: segmentation; unemployment; exclusion.

\title{
Introducción
}

El mercado de trabajo argentino presenta características propias de un esquema segmentario, en el cual mientras unos trabajadores acceden a puestos de trabajo registrados y con la debida protección de derechos asociada al trabajo, otros no transitan la misma suerte, ya sea por encontrarse en empleos inestables, no registrados y con su consecuente pérdida de derechos o simplemente por ubicarse al margen del trabajo, sin lograr una exitosa inserción laboral.

El fin que se propone este documento radica en proporcionar evidencia que permita aproximarse al escenario laboral acontecido entre 2004 y 2012 en Argentina. Se pretende en particular destacar que aun teniendo en cuenta que, a pesar de constituir una fase de notables logros a nivel macroeconómico, persisten déficits ligados a la disponibilidad de puestos de trabajo en general y de aquellos protegidos en particular. Por lo tanto, en la diagramación y presentación de la información se otorga énfasis a la caracterización de los trabajadores con mayores niveles de precariedad. Se estiman la incidencia y evolución en el tiempo de ciertas variables clave que caracterizan a los individuos en edad activa que participan de la Población Económicamente Activa de 18 a 59 años.

2

María Eugenia Sconfienza

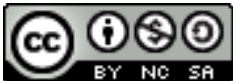

Revista Economía y Sociedad by Universidad Nacional is licensed under a CreativeCommons Reconocimiento-NoComercial- 


\section{Algunas características conceptuales e indicadores sociolaborales seleccionados de Argentina}

En este desarrollo de carácter descriptivo se detalla la evolución y características de algunos de los principales indicadores relacionados con el mercado de trabajo argentino, a la vez que se mencionan ciertos encuadres teóricos que resultan útiles para la interpretación de los resultados empíricos.

Así, este escrito se encuentra dividido en dos secciones. En la primera se desarrolla una breve descripción acerca de ciertas nociones teóricas que se ajustan al mercado de trabajo argentino. Mientras que en la segunda sección se describen algunos de los principales indicadores que dan cuenta de la evolución del mercado laboral argentino entre los años 2004 a 2012 -cuando la economía argentina registró una fase de crecimiento económico- con el propósito de dimensionar y cuantificar la fisonomía que cobran la segmentación y precarización laboral en el país.

La información cuantitativa utilizada proviene mayoritariamente de la Encuesta Permanente de Hogares (EPH) realizada por el Instituto Nacional de Estadística y Censos (INDEC) ${ }^{2}$. Con las bases de microdatos de esta encuesta se realizaron indagaciones descriptivas vía procesamientos estadísticos en sintonía con los objetivos propuestos en la investigación.

\section{Precariedad, segmentación y exclusión laboral}

El mercado de trabajo en Argentina presenta características que lo identifican como un compuesto de más de un segmento, en donde se tiene en cuenta que el volumen y la velocidad de creación de puestos de trabajo no resultan suficientes para incluir a la oferta laboral disponible o potencialmente disponible. Empleadores y trabajadores se desenvuelven en un contexto de segmentación a la vez que se constituyen como protagonistas de esta condición.

Tal como lo plantea la teoría de la segmentación (Piore, 1983), existe una coincidencia entre el entorno social de los trabajadores -clases- y las divisiones del mercado de trabajo. Los puestos

\footnotetext{
${ }^{2}$ La EPH es una encuesta cuyo universo de estudio es la población urbana residente en viviendas particulares de la Argentina que vive en ciudades de más de 100.000 habitantes y en capitales de provincias. Se trata de una encuesta por muestreo, en la cual las viviendas son seleccionadas a través de un esquema de tipo probabilístico, estratificado y polietápico. El registro de la información se realiza a través de un cuestionario en papel administrado en una entrevista directa. Si bien la periodicidad de los resultados es trimestral, cada vivienda tiene una sola semana asignada en ese período en la cual contesta los cuestionarios. Cada vivienda es visitada cuatro veces a lo largo de 18 meses: dos trimestres consecutivos es entrevistada, los dos siguientes sale de la muestra y los dos restantes vuelve a ser entrevistada. La misma, abarca a cerca del 70\% del total de la población urbana (a partir del tercer trimestre de 2006, aumenta el tamaño muestral alcanzando un total de 31 aglomerados, lo que implica una muestra trimestral de 26.000 viviendas).
}

María Eugenia Sconfienza

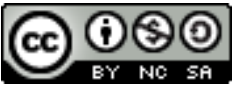

Revista Economía y Sociedad by Universidad Nacional is licensed under a CreativeCommons Reconocimiento-NoComercialCompartirlgual 4.0 Internacional License. Creado a partir de la obra en http://www.revistas.una.ac.cr/index.php/economia 
de trabajo inestables, de salarios más bajos, sin acceso a la seguridad social y por tanto en condiciones de mayor precariedad -sector secundario- así como el desempleo, se concentran en las clases bajas de la población. Los estratos más pobres y de niveles educativos más bajos de la población difícilmente acceden a puestos de trabajo formales. Este tipo de teorías parten de la premisa que todos aquellos que participan del mercado laboral desean mejores empleos, los que solo se encuentran disponibles para una fracción de la fuerza laboral. Por tanto, quienes no acceden a estos buenos empleos, toman "malos" trabajos o permanecen desempleados configurando un excedente laboral en el cual prevalecen el autoempleo de subsistencia y el desempleo estructural; conformando sectores de la sociedad que devienen en innecesarios y por tanto prescindibles -asimilables a lo que el marxismo denominó ejercito industrial de reserva (Nun, 1969) en estrecha asociación con el concepto de marginalidad (BennholdtThomsen, 1981).

Es así, que la constitución de la estructura social argentina muestra la existencia de diferencias salariales en favor de quienes logran emplearse en puestos de trabajo registrados respecto de los asalariados en puestos no registrados en la seguridad social ${ }^{3}$. Aquí se entienden como empleos del sector "primario", aquellos empleos estables y registrados, caracterizados por una duración de tiempo indeterminado de acuerdo a la normativa laboral vigente, insertos en un convenio colectivo con garantías legales de estabilidad contra despidos sin causa, protegidos por el sistema de seguridad social, con salario indirecto para cubrir el cuidado de la salud, con mecanismos de previsión social, asignaciones familiares, protección contra los riesgos del trabajo y seguro contra el desempleo, entre otros. En oposición, se ubican los hogares signados por la exclusión y marginalidad que encuentran sus recursos laborales profundamente deteriorados. Estos recursos a su vez son difícilmente recuperables ya que las unidades domésticas necesitan indefectiblemente ingresos para activar otros recursos tales como el autoempleo, la propia provisión de bienes y servicios, y la pequeña producción doméstica. En estos mercados segmentados donde prevalecen amplios sectores de trabajadores de bajos recursos, es que la informalidad y la exclusión se presentan como situaciones preponderantes y persistentes. Son entornos en los cuales el mercado laboral segmentado se traduce en desigualdad real (Häusermann, S. y Schwander, H., 2010).

Siguiendo a Castel (2010) quien Ilama la atención sobre los excluidos de la sociedad salarial, puede sostenerse que en Argentina un contingente relevante de trabajadores carece de las condiciones necesarias para ser reconocidos como trabajadores de pleno derecho. Es decir, personas que hasta, inclusive, trabajando, se encuentran inmersas en condiciones de pobreza ${ }^{4}$.

\footnotetext{
${ }^{3}$ Véanse al respecto entre otros, Groisman (2013) y Groisman, Beccaria y Monsalvo (2006).

${ }^{4}$ En Argentina existen algunos estudios que focalizan su atención en el conjunto de los denominados "trabajadores pobres". Véase entre otros Calero y Sorokin (2013), quienes identifican -para luego analizar- a este grupo, como aquellos que han participado en el mercado laboral al menos una hora en la semana de referencia pero que a su vez habitan en hogares cuyos ingresos son inferiores a un umbral mínimo determinado.
} 4

María Eugenia Sconfienza

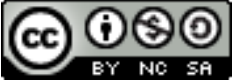

Revista Economía y Sociedad by Universidad Nacional is licensed under a CreativeCommons Reconocimiento-NoComercialCompartirlgual 4.0 Internacional License. Creado a partir de la obra en http://www.revistas.una.ac.cr/index.php/economia 
Fenómeno que a su vez se relaciona en la mayoría de los casos, a una estructura socioeconómica que presenta una limitada movilidad laboral intergeneracional. De esta forma, nociones como la de precariedad laboral, segmentación y exclusión se relacionan dando cuenta de la realidad de un universo de trabajadores con transitan trayectorias laborales signadas por la ausencia de estabilidad (intermitencia laboral) y el no registro ${ }^{5}$, y situación de exclusión del mercado de trabajo en las que no logran activarse independientemente de sus esfuerzos ${ }^{6}$. Ello condensa una lógica de sociedad segmentada en la cual conviven trabajadores con pleno derecho y otros marginados de estas posibilidades.

Robert Castel (1997) destaca que cuanto más significativa es la precariedad de la situación del trabajador respecto del empleo, mayores son los riesgos de rupturas sociales y familiares, de reducción de la sociabilidad, de pérdida de identidad, de sufrimiento psíquico y mental, de empobrecimiento de los ingresos y por tanto, mayor el deterioro de las condiciones de vida. En este sentido, a medida que se perpetúan las manifestaciones de precariedad laboral, mayores las probabilidades de profundizar y reforzar la situación de exclusión. Ahora bien, ¿cómo se presenta esta estructura segmentada para el caso de Argentina?

Los datos para Argentina evidencian un período que ha presentado una lenta recuperación desde 2003 debido a la intensidad de la crisis acontecida en 2001/2002, que llevaron a una proporción importante de la población a situaciones de pobreza e indigencia, así como pauperización de su situación laboral. Según los datos proporcionados por el INDEC, las tasas de desempleo eran superiores a un $20 \%-21,5 \%$ en mayo de 2002 - y las tasas de pobreza oscilaban en un $50 \%$. La lenta recuperación económica, la implementación de programas de protección social y la creación de empleo posteriores fueron reduciendo los peores indicadores económico-sociales llevando a una mejora en los ingresos reales de los trabajadores.

Suelen destacarse algunos rasgos del contexto económico contrastantes respecto del período precedente -década de 1990- los cuales habrían favorecido la mejoría de ciertos indicadores económicos y laborales: tipo de cambio real alto; mejora de la competitividad de la economía; crecimiento con creación de empleo; reversión en privatizaciones y desregulación; incremento en la formalización laboral; rol central y protagónico de instituciones laborales como la negociación colectiva, el Salario Mínimo Vital y Móvil -SMVM-, y la inspección laboral; y caída de la desigualdad; entre otros (Bertranou, Casanova, Jiménez y Jiménez, 2013).

\footnotetext{
${ }^{5}$ El empleo registrado representa la instancia que mejor asegura derechos sociales y ciudadanos en base a la contribución productiva del trabajador en relación de dependencia. Vale decir, que si bien la exclusión puede responder a múltiples causas, la limitación al acceso al mercado de trabajo o una inserción laboral endeble, actúan como potenciadores de esta situación de exclusión. A estos trabajadores outsiders lo que los define es su trayectoria ocupacional más que su situación laboral en un momento determinado (Hausermann y Schwander, 2010)

${ }^{6}$ Para un mayor detalle acerca de las diversas corrientes teóricas relativas a los conceptos de mercado laboral segmentado así como exclusión, precariedad e informalidad, véanse Fernández Huerga (2010) y Sconfienza (2016).
} 
Sin embargo, las mejoras en términos laborales no se advirtieron más intensas para los hogares con menores recursos -cuyos jefes no tiene educación secundaria completa-. Ello constituye un primer indicio acerca de las características distributivas que tuvo esta fase expansiva, especialmente luego de superar los niveles críticos en los dos años posteriores a la crisis. En efecto, solo en este breve período la mejora en los ingresos de los hogares se mostró más intensa para las familias con menores recursos. Luego, el crecimiento del empleo fue diferenciándose según nivel educativo: más fuerte para quienes tenían mayor nivel educativo. Otro rasgo que prevaleció en el período fue una elevada inestabilidad laboral (Groisman, 2010). La significativa proporción de empleos precarizados en la estructura de empleo conlleva una recurrente rotación laboral consecuencia de un mercado laboral que se presenta como inestable para un segmento de la población. Este, si bien fue un rasgo que se extendió en forma muy marcada en la sociedad argentina durante el decenio de los noventa, especialmente en la segunda mitad del mismo (Groisman y Beccaria, 2009), en los años que siguieron al 2001 continuó -con una intensidad menor-, pero se puede afirmar que siguió afectando especialmente a los hogares de menores recursos.

\section{Un mercado laboral segmentado: la evidencia argentina}

Desde mediados de 2003 se inicia un período en el cual el patrón de crecimiento se denominó de desarrollo inclusivo (Zuazúa, 2006). Fue un período de intensiva aplicación de programas y políticas socioeconómicas que inyectaron recursos en los sectores más vulnerados (Sconfienza, 2016).

La economía argentina registró una fase de crecimiento económico entre 2004 y 2012 con tasas de crecimiento del PBI que oscilaron en torno al 8-9\%, con la excepción de los períodos 2008, 2009 y 2012. Estudios disponibles dan cuenta de este período de bonanza en términos económicos y consecuentemente laborales en el período 2004-2012, al mismo tiempo que distinguen dos etapas. Una primera que abarca desde 2003 a 2007/2008, en la cual se percibe un crecimiento que condice con una utilización de mano de obra más intensiva, de mayor evidencia de una elevada elasticidad empleo-producto. Mientras, que una segunda, a partir de 2007/2008 da muestras de un estancamiento de la fuerza de trabajo (Azpiazu y Schorr, 2010), reflejo en parte del coletazo de la crisis del mercado de hipotecas sub-prime en Estados Unidos acontecida en agosto de 2007.

Se observa una significativa disminución del ritmo de crecimiento del Producto Bruto Interno (PBI) en 2008 (Tabla 1), manifestada como consecuencia en la merma de inversión y consumo privado (Arceo, González, Mendizábal, y Basualdo, 2010). Asimismo, se argumenta que el crecimiento en la industria al mismo tiempo que logró cierta recuperación perdida en el período 1976-2001, no implicó una ruptura o modificaciones abruptas en relación con años previos -patrón de inserción internacional asociado a recursos naturales y algunas industrias productoras de commodities-. Se reconoce a la vez la persistencia de un patrón de 6

María Eugenia Sconfienza

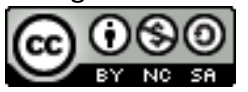

Revista Economía y Sociedad by Universidad Nacional is licensed under a CreativeCommons Reconocimiento-NoComercialCompartirlgual 4.0 Internacional License. Creado a partir de la obra en http://www.revistas.una.ac.cr/index.php/economia 
concentración del capital en el cual la redistribución de ingresos se mostró en favor de las fracciones más concentradas y transnacionalizadas del capital (Azpiazu y Schorr, 2010).

Tabla 1.

Variación anual (\%) del Producto Interno Bruto real (PIB) 2004-2012

\begin{tabular}{llllllllll}
\hline & $\mathbf{2 0 0 4}$ & $\mathbf{2 0 0 5}$ & $\mathbf{2 0 0 6}$ & $\mathbf{2 0 0 7}$ & $\mathbf{2 0 0 8}$ & $\mathbf{2 0 0 9}$ & $\mathbf{2 0 1 0}$ & $\mathbf{2 0 1 1}$ & $\mathbf{2 0 1 2}$ \\
\hline PIB real & $9,0 \%$ & $9,2 \%$ & $8,4 \%$ & $8,0 \%$ & $3,1 \%$ & $0,1 \%$ & $9,5 \%$ & $8,4 \%$ & $0,8 \%$ \\
\hline
\end{tabular}

Fuente: elaboración propia con base en datos de la Dirección Nacional de Cuentas Nacionales - INDEC (2004-2012)

Durante el período bajo análisis, la industria evidenció etapas de diversa intensidad. Entre los años 2004 y 2012, con excepción de 2009 debido al impacto de la crisis internacional, la industria ha crecido en forma continua, dinámica que se estancaría hacia el año 2012. El crecimiento sostenido del sector se corresponde con un incremento del consumo en los hogares a raíz de la recuperación económica, con medidas entre otras como la promoción a la sustitución de importaciones -incentivos a la inversión como exenciones impositivas, restricción de importaciones ${ }^{7}$, políticas salariales de incrementos del Salario Mínimo, Vital y Móvil (SMVM), moratoria previsional y políticas de transferencia de ingresos como la Asignación Universal por Hijo -AUH-. En el año 2009 con el propósito de sustituir importaciones, se implementaron algunas licencias no automáticas para productos importados que podían ser producidos localmente. Desde el año 2010 estas políticas se profundizaron mediante medidas como obligaciones de compensación de importaciones con exportaciones, algunos obstáculos burocráticos a la aprobación de importaciones y prohibiciones directas.

Según datos proporcionados por el Ministerio de Industria en base al INDEC, la evolución de exportaciones de manufactura de origen industrial entre los años 2003 y 2012 pasó de 8.000 millones de dólares a 27.500 millones de dólares. No obstante, no resulta posible atribuir a este crecimiento un cambio estructural a favor de la industria manufacturera.

Si bien se detuvo la tendencia a la desindustrialización que caracterizó al país en las últimas décadas del siglo $\mathrm{XX}$, el crecimiento del sector no resultó superior en términos relativos al de otros sectores económicos (Marshall y Perelman, 2013). En resumen, la evolución de este sector, aunque remarcable, no habría repercutido en un cambio de modelo productivo de país, en una utilización intensiva de mano de obra, ni en un profundo crecimiento económico generalizado.

\footnotetext{
7 Indumentaria, maquinaria industrial, electrodomésticos -heladeras, lavarropas-, calzado, juguetes, tecnología -celulares, televisores, equipos e instrumentos de audio-, productos de cuero, cosechadoras, bicicletas, motos.
} 


\section{Caracterización laboral de la población}

La desocupación para el total de la población en edad activa de 18 a 59 años evidenció un descenso de algo más de 4 p.p. en el período bajo análisis -2004 a 2012-, pero si se observa en particular la última fase 2008-2012, se puede apreciar que la tasa de desocupación se mantuvo constante.

En términos de género, en todo el período se mantuvo la proporcionalidad de mayor desocupación relativa de las mujeres respecto de la de los varones en más de 3 p.p., de lo que se deduce que en su conjunto las mujeres sufren más la desocupación que los varones. Tendencia que se mantiene y perpetúa en el tiempo (Tabla 2).

Tabla 2.

Condición de actividad y tasa de desocupación. Total 18 a 59 años. 2004-2008-2012 (\%)

\begin{tabular}{lccc}
\hline & $\mathbf{2 0 0 4}$ & $\mathbf{2 0 0 8}$ & $\mathbf{2 0 1 2}$ \\
\hline Total & & & \\
Activos & 75,6 & 74,8 & 74,3 \\
Inactivos & 24,4 & 25,2 & 25,7 \\
Total & 100,0 & 100,0 & 100,0 \\
Desocupados & 11,7 & 7,3 & 7,1 \\
Varones & & & \\
Activos & 88,9 & 87,9 & 87,8 \\
Inactivos & 11,1 & 12,1 & 12,2 \\
Total & 100,0 & 100,0 & 100,0 \\
Desocupados & 10,3 & 5,9 & 5,7 \\
Mujeres & & & \\
Activos & 63,2 & 62,7 & 61,6 \\
Inactivos & 36,8 & 37,3 & 38,4 \\
Total & 100,0 & 100,0 & 100,0 \\
Desocupados & 13,7 & 9,0 & 9,0 \\
\hline
\end{tabular}

Fuente: elaboración propia con base en EPH - INDEC (IV trimestres)

En el período 2004-2012, el peso relativo de asalariados registrados se incrementó considerablemente y el de no registrados se redujo (Tabla 4), aunque cabe distinguir esta evolución en dos etapas. Por una parte, los trabajadores registrados se incrementaron en un $36,8 \%$ en 2008 respecto de 2004 -el incremento notable en el grupo de asalariados registrados se debe en parte a continuas políticas por buscar frenar la informalidad, pero también al crecimiento económico del período, razón por la cual el marcado incremento de trabajadores registrados se concentra en particular entre los años 2004 y 2008-, relación que en 2012

8

María Eugenia Sconfienza

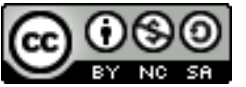

Revista Economía y Sociedad by Universidad Nacional is licensed under a CreativeCommons Reconocimiento-NoComercial- 
respecto de 2008 bajó a menos de un tercio, 12\%. Las demás categorías ocupacionales se mantuvieron constantes (Tabla 3). Ello estaría indicando que en términos absolutos no se registró una reducción del no registro. Es decir, es probable que se creara empleo formal al mismo tiempo que persistieran empleos revistiendo la categoría de no registro (Sconfienza, 2016). Si en cambio se observa la composición porcentual de los ocupados según categoría ocupacional los registrados pasan a ser algo más de la mitad en 2012, y los no registrados descienden a poco más de un cuarto (Tabla 4). Cabe destacar que entre 2004 y 2012 el registro se mostró con mayor frecuencia en el caso de las mujeres -15\%-, mientras que para el grupo de los varones el mismo registró un incremento de 11 p.p. (Tabla 4).

Tabla 3.

Insumo de mano de obra según categoría ocupacional 2004-2008-2012 (en puestos de trabajo)

\begin{tabular}{lccc}
\hline & $\mathbf{2 0 0 4}$ & $\mathbf{2 0 0 8}$ & $\mathbf{2 0 1 2}$ \\
\hline Asalariados registrados & 6.567 .319 & 8.981 .153 & 10.054 .750 \\
Asalariados no registrados & 4.773 .007 & 4.524 .579 & 4.629 .312 \\
Cuenta propia & 2.285 .979 & 2.584 .396 & 2.689 .910 \\
Patrones & 1.601 .156 & 1.690 .383 & 1.740 .258 \\
Familiares No remunerados & 288.424 & 245.761 & 242.931 \\
\hline
\end{tabular}

Fuente: elaboración propia con base en la Dirección Nacional de Cuentas Nacionales - INDEC (2004-2012)

Tabla 4.

Categoría ocupacional. Total ocupados 18 a 59 años. 2004-2008-2012 (\%)

\begin{tabular}{lccc}
\hline & $\mathbf{2 0 0 4}$ & $\mathbf{2 0 0 8}$ & $\mathbf{2 0 1 2}$ \\
\hline Total & & & \\
Asalariados registrados & 39,9 & 49,8 & 52,7 \\
Asalariados no registrados & 36,6 & 28,6 & 26,5 \\
Patrón & 4,0 & 4,4 & 3,9 \\
Cuenta propia & 18,6 & 16,5 & 16,5 \\
Trabajador familiar sin remuneración & 0,9 & 0,6 & 0,4 \\
Total & 100,0 & 100,0 & 100,0 \\
Varones & & & \\
Asalariados registrados & 41,7 & 51,3 & 52,8 \\
Asalariados no registrados & 32,3 & 24,7 & 24,0 \\
Patrón & 5,1 & 5,4 & 4,6 \\
Cuenta propia & 20,6 & 18,3 & 18,4 \\
Trabajador familiar sin remuneración & 0,3 & 0,3 & 0,2 \\
Total & 100,0 & 100,0 & 100,0 \\
\hline
\end{tabular}




\begin{tabular}{lccc}
\hline & $\mathbf{2 0 0 4}$ & $\mathbf{2 0 0 8}$ & $\mathbf{2 0 1 2}$ \\
\hline Mujeres & & & \\
Asalariados registrados & 37,4 & 47,9 & 52,6 \\
Asalariados no registrados & 42,5 & 33,7 & 30,0 \\
Patrón & 2,5 & 3,1 & 3,0 \\
Cuenta propia & 15,9 & 14,1 & 13,9 \\
Trabajador familiar sin remuneración & 1,8 & 1,1 & 0,6 \\
Total & 100,0 & 100,0 & 100,0 \\
\hline
\end{tabular}

Nota: Para el caso de patrones y trabajadores familiares no remunerados existe la posibilidad de un sesgo debido al potencial error derivado de la magnitud acotada de la muestra proporcionada por la EPH.

Fuente: elaboración propia con base en EPH - INDEC (IV trimestres)

Algunas de las variables de análisis de interés que permiten comprender la estructura ocupacional que caracteriza al mercado laboral argentino, son por ejemplo el nivel educativo, el nivel de calificación, el tamaño de los establecimientos de trabajo, las ramas de actividad y el nivel de ingresos. Las mismas se describen y analizan a continuación.

\section{Nivel educativo}

En términos generales, la evolución de la ocupación acompaña el nivel educativo de la población. Es decir, se amplía el volumen de los niveles más altos en el universo de ocupados, como corolario del incremento de la escolarización de la población en general. No obstante, al comparar el nivel educativo del total de la población respecto de la población ocupada, se evidencia que si bien no es determinante, sí existe una correlación entre el nivel educativo y la condición de ocupación, particularmente en el caso de las mujeres. A continuación se analizan algunas particularidades interesantes que salen a la luz al observar la evolución de los indicadores educativos con mayor precisión en función del género.

Respecto de la población masculina, la evolución muestra que en el período 2004-2012 no se presentaban variaciones significativas entre la estructura educativa del total de la población y el total de ocupados. Pero, se puede apreciar que para el caso de las mujeres, el mayor nivel educativo relativo se concentra en la población ocupada. Las cifras indican que el total de mujeres con nivel educativo alto superior incompleto/completo por una parte creció significativamente entre 2004 y 2012, pasando de 55,2\% a 63,4\% -tendencia que también se observa en el caso de las varones-, pero por otra parte, estos niveles educativos en 2012 representaban el $71,2 \%$ de la fuerza ocupada femenina -mientras que para el caso de los varones esta cifra era de 57,9\%-. Como consecuencia, se hace visible que en la fuerza laboral masculina a diferencia de la femenina la educación presenta un peso relativo menor. (Tabla 5).

10

María Eugenia Sconfienza

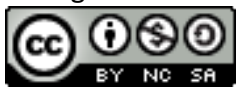

Revista Economía y Sociedad by Universidad Nacional is licensed under a CreativeCommons Reconocimiento-NoComercialCompartirlgual 4.0 Internacional License.

Creado a partir de la obra en http://www.revistas.una.ac.cr/index.php/economia 
Tabla 5.

Nivel educativo. Población total y ocupada. 18 a 59 años. 2004-2008-2012 (\%)

\begin{tabular}{lcccccc}
\hline & \multicolumn{3}{c}{ Total } & \multicolumn{3}{c}{ Ocupados } \\
\cline { 2 - 7 } & $\mathbf{2 0 0 4}$ & $\mathbf{2 0 0 8}$ & $\mathbf{2 0 1 2}$ & $\mathbf{2 0 0 4}$ & $\mathbf{2 0 0 8}$ & $\mathbf{2 0 1 2}$ \\
\hline Total & & & & & & \\
Hasta primario incompleto & 6,8 & 6,2 & 4,4 & 6,0 & 5,2 & 3,5 \\
Hasta secundario incompleto & 40,9 & 37,3 & 35,3 & 39,7 & 34,9 & 33,0 \\
Hasta superior incompleto & 38,3 & 40,4 & 42,8 & 36,3 & 39,2 & 41,1 \\
Superior completo & 14,0 & 16,2 & 17,5 & 18,0 & 20,8 & 22,3 \\
Total & 100,0 & 100,0 & 100,0 & 100,0 & 100,0 & 100,0 \\
Varones & & & & & & \\
Hasta primario incompleto & 6,9 & 6,5 & 4,6 & 6,3 & 5,9 & 4,0 \\
Hasta secundario incompleto & 43,8 & 40,3 & 38,5 & 44,7 & 40,1 & 38,1 \\
Hasta superior incompleto & 37,8 & 40,3 & 43,3 & 35,8 & 39,2 & 42,3 \\
Superior completo & 11,4 & 13,0 & 13,6 & 13,3 & 14,8 & 15,6 \\
Total & 100,0 & 100,0 & 100,0 & 100,0 & 100,0 & 100,0 \\
Mujeres & & & & & & \\
Hasta primario incompleto & 6,6 & 5,9 & 4,2 & 5,7 & 4,2 & 2,9 \\
Hasta secundario incompleto & 38,2 & 34,5 & 32,3 & 33,0 & 27,8 & 25,8 \\
Hasta superior incompleto & 38,8 & 40,5 & 42,2 & 37,0 & 39,1 & 39,5 \\
Superior completo & 16,4 & 19,1 & 21,2 & 24,3 & 28,8 & 31,7 \\
Total & 100,0 & 100,0 & 100,0 & 100,0 & 100,0 & 100,0 \\
\hline
\end{tabular}

Fuente: elaboración propia con base en EPH - INDEC (IV trimestres)

Ahora bien, al analizar la composición de los ocupados en función de su nivel educativo y la categoría ocupacional se puede ver que si bien en el período 2004-2012, se observa un incremento generalizado del nivel educativo en todas las categorías, aquellas que reconcentran los niveles educativos más bajos son las ocupaciones ligadas al cuentapropismo y a los puestos de trabajo asalariados no registrados (Figura 1).

La marcada diferencia entre géneros se observa cuando para ser asalariado registrado en el caso de la mujer a comparación del varón los requisitos educativos parecieran ser más selectivos. Del total de asalariados registrados en 2012 , un $67,7 \%$ se compone de ocupados con nivel de superior completo/incompleto para el caso de los varones. No obstante, esa proporción asciende a 85,3\% en el caso de las mujeres (Tabla 6). 


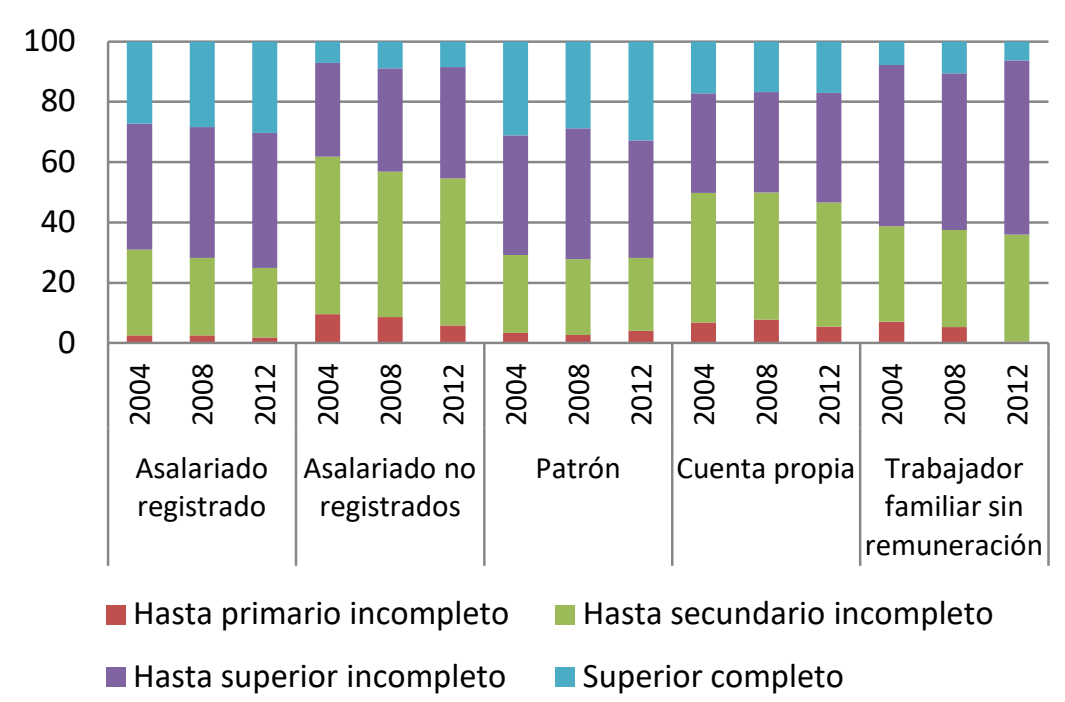

Figura 1. Nivel educativo según categoría ocupacional. 18 a 59 años. 2004-2008-2012 (\%). Fuente: elaboración propia con base en EPH - INDEC (IV trimestres)

Tabla 6.

Nivel educativo según categoría ocupacional. Varones y mujeres 18 a 59 años. 2004-2008-2012 (\%)

\begin{tabular}{|c|c|c|c|c|c|c|c|c|c|c|c|c|}
\hline & \multicolumn{3}{|c|}{ Asalariado registrado } & \multicolumn{3}{|c|}{$\begin{array}{l}\text { Asalariado no } \\
\text { registrados }\end{array}$} & \multicolumn{3}{|c|}{ Patrón } & \multicolumn{3}{|c|}{ Cuentapropia } \\
\hline & 2004 & 2008 & 2012 & 2004 & 2008 & 2012 & 2004 & 2008 & 2012 & 2004 & 2008 & 2012 \\
\hline \multicolumn{13}{|l|}{ Varones } \\
\hline $\begin{array}{l}\text { Hasta primario } \\
\text { incompleto }\end{array}$ & 3,4 & 3,1 & 2,2 & 9,4 & 9,3 & 6,0 & 3,9 & 2,8 & 5,8 & 7,7 & 10,0 & 6,1 \\
\hline $\begin{array}{l}\text { Hasta secundario } \\
\text { incompleto }\end{array}$ & 36,5 & 33,3 & 30,0 & 54,9 & 53,0 & 51,8 & 27,0 & 29,4 & 24,9 & 49,9 & 45,3 & 46,9 \\
\hline $\begin{array}{l}\text { Hasta superior } \\
\text { incompleto }\end{array}$ & 42,1 & 44,8 & 47,6 & 30,1 & 31,7 & 35,8 & 40,8 & 42,8 & 41,8 & 30,2 & 32,1 & 35,3 \\
\hline Superior completo & 18,0 & 18,8 & 20,1 & 5,6 & 6,0 & 6,4 & 28,3 & 25,0 & 27,5 & 12,2 & 12,7 & 11,7 \\
\hline $\begin{array}{l}\text { Total } \\
\text { Mujeres }\end{array}$ & 100 & 100 & 100 & 100 & 100 & 100 & 100 & 100 & 100 & 100 & 100 & 100 \\
\hline $\begin{array}{l}\text { Hasta primario } \\
\text { incompleto }\end{array}$ & 1,3 & 1,8 & 1,2 & 9,8 & 7,9 & 5,6 & 1,7 & 2,6 & 0,3 & 5,4 & 3,8 & 4,3 \\
\hline $\begin{array}{l}\text { Hasta secundario } \\
\text { incompleto }\end{array}$ & 16,3 & 14,6 & 13,5 & 49,3 & 43,7 & 45,4 & 22,6 & 15,1 & 22,7 & 31,0 & 36,8 & 30,4 \\
\hline $\begin{array}{l}\text { Hasta superior } \\
\text { incompleto }\end{array}$ & 41,3 & 41,5 & 40,9 & 32,3 & 36,6 & 38,1 & 36,4 & 44,6 & 32,7 & 37,8 & 35,5 & 38,3 \\
\hline Superior completo & 41,1 & 42,0 & 44,4 & 8,6 & 11,9 & 10,9 & 39,3 & 37,7 & 44,2 & 25,9 & 23,8 & 27,0 \\
\hline Total & 100 & 100 & 100 & 100 & 100 & 100 & 100 & 100 & 100 & 100 & 100 & 100 \\
\hline
\end{tabular}

Nota: se excluye a los trabajadores familiares

Fuente: elaboración propia con base en EPH - INDEC (IV trimestres)

12 
Podría pensarse que la información presentada aquí abona dos perspectivas, por un lado que quienes detentan un empleo logran acceder a mayores niveles educativos y por el otro que quienes poseen mayores niveles educativos, se encuentran más proclives a obtener un empleo. La teoría del capital humano indica que a mayor capacitación mayor probabilidad de ocupación. No obstante, este razonamiento deja afuera la problemática asociada a las limitaciones de la demanda de empleo existente. Con lo cual se puede argüir que en contextos como el argentino, donde los puestos de empleo disponibles resultan insuficientes frente a la demanda real -y donde las mujeres presentan mayores tasas relativas de desempleo respecto de los varones-, los empleadores tienden a priorizar a quienes disponen de mayor nivel educativo, lo que no necesariamente implica que el nivel educativo requerido siempre se corresponda con las tareas del puesto de trabajo ${ }^{8}$. Bajo esta misma lógica, quienes conforman el universo de ocupados encuentran que los empleos de mejor calidad se reservan para aquellos colectivos de mayor nivel educativo.

\section{Calificación de los puestos de trabajo}

Uno de los indicadores más relevantes es el que explica la distribución del nivel de registro asociado de los distintos niveles de calificación.

En términos generales, las categorías más beneficiadas por el registro se concentraron primero en el conjunto de los no calificados. Categoría en la que el registro subió 17,3 p.p. entre 2004 y $2014-18,6$ p.p. para el caso de los varones y 16,4 p.p. para las mujeres-. Nuevamente, vale destacar que el salto significativo se registró en el período 2004-2008 (Tabla 7).

Tabla 7.

Categoría ocupacional según nivel de calificación. 18 a 59 años. 2004-2008-2012 (\%)

\begin{tabular}{lllllllllllll}
\hline & \multicolumn{3}{c}{ Profesionales } & \multicolumn{3}{c}{ Técnicos } & \multicolumn{3}{c}{ Operativos } & \multicolumn{3}{c}{ No calificados } \\
\cline { 2 - 13 } & $\mathbf{2 0 0 4}$ & $\mathbf{2 0 0 8}$ & $\mathbf{2 0 1 2}$ & $\mathbf{2 0 0 4}$ & $\mathbf{2 0 0 8}$ & $\mathbf{2 0 1 2}$ & $\mathbf{2 0 0 4}$ & $\mathbf{2 0 0 8}$ & $\mathbf{2 0 1 2}$ & $\mathbf{2 0 0 4}$ & $\mathbf{2 0 0 8}$ & $\mathbf{2 0 1 2}$ \\
\hline Total & & & & & & & & & & & & \\
Asalariado registrado & 44,9 & 55,0 & 57,5 & 56,9 & 60,3 & 62,1 & 42,2 & 51,8 & 54,2 & 21,9 & 34,6 & 39,2 \\
Asalariado no & 15,4 & 11,4 & 10,2 & 13,8 & 11,5 & 11,3 & 32,0 & 24,6 & 22,5 & 69,0 & 58,9 & 56,7 \\
registrado & 18,6 & 17,2 & 15,1 & 13,8 & 15,1 & 14,1 & 0,0 & 0,0 & 0,0 & 0,0 & 0,0 & 0,0 \\
Patrón & 21,0 & 16,4 & 17,2 & 15,4 & 13,1 & 12,6 & 25,3 & 23,1 & 23,1 & 6,6 & 4,9 & 2,8 \\
Cuenta propia & 0,2 & 0,0 & 0,0 & 0,1 & 0,1 & 0,0 & 0,6 & 0,5 & 0,2 & 2,5 & 1,6 & 1,2 \\
Trabajador familiar & sin remuneración & & & & & & & & & & &
\end{tabular}

\footnotetext{
${ }^{8}$ En relación a las disparidades entre género, cabe destacar como se expuso precedentemente que la estructura de los empleos existentes permite ver que se da una tasa de empleo mayor para mujeres profesionales.
}

María Eugenia Sconfienza

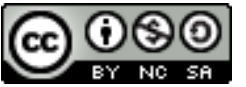

Revista Economía y Sociedad by Universidad Nacional is licensed under a CreativeCommons Reconocimiento-NoComercial- 


\begin{tabular}{llllllllllllll}
\hline & \multicolumn{1}{c}{ Profesionales } & \multicolumn{3}{c}{ Técnicos } & \multicolumn{3}{c}{ Operativos } & \multicolumn{3}{c}{ No calificados } \\
\cline { 2 - 13 } & $\mathbf{2 0 0 4}$ & $\mathbf{2 0 0 8}$ & $\mathbf{2 0 1 2}$ & $\mathbf{2 0 0 4}$ & $\mathbf{2 0 0 8}$ & $\mathbf{2 0 1 2}$ & $\mathbf{2 0 0 4}$ & $\mathbf{2 0 0 8}$ & $\mathbf{2 0 1 2}$ & $\mathbf{2 0 0 4}$ & $\mathbf{2 0 0 8}$ & $\mathbf{2 0 1 2}$ \\
\hline $\begin{array}{l}\text { Total } \\
\text { Varones }\end{array}$ & 100 & 100 & 100 & 100 & 100 & 100 & 100 & 100 & 100 & 100 & 100 & 100 \\
Asalariado registrado & 46,2 & 55,3 & 58,5 & 52,0 & 52,0 & 52,1 & 43,6 & 52,3 & 53,6 & 27,5 & 44,1 & 46,1 \\
Asalariado no & 12,4 & 8,1 & 8,2 & 10,8 & 10,8 & 11,1 & 31,0 & 25,4 & 24,2 & 60,6 & 46,3 & 45,8 \\
registrado & 23,9 & 21,8 & 19,1 & 21,0 & 21,0 & 20,4 & 0,0 & 0,0 & 0,0 & 0,0 & 0,0 & 0,0 \\
$\begin{array}{l}\text { Patrón } \\
\text { Cuenta propia }\end{array}$ & 17,5 & 14,8 & 14,0 & 16,2 & 16,2 & 16,4 & 25,2 & 22,1 & 22,2 & 10,9 & 8,6 & 7,2 \\
$\begin{array}{l}\text { Trabajador familiar } \\
\text { sin remuneración }\end{array}$ & 0,0 & 0,0 & 0,1 & 0,0 & 0,0 & 0,0 & 0,2 & 0,2 & 0,0 & 1,1 & 0,9 & 0,9 \\
Total & 100 & 100 & 100 & 100 & 100 & 100 & 100 & 100 & 100 & 100 & 100 & 100 \\
Mujeres & & & & & & & & & & & & \\
Asalariado registrado & 43,2 & 64,4 & 56,3 & 72,0 & 72,0 & 71,1 & 39,4 & 60,8 & 55,8 & 17,5 & 46,0 & 33,9 \\
Asalariado no & 19,3 & 0,4 & 12,4 & 8,3 & 8,3 & 11,4 & 33,8 & 7,8 & 18,5 & 75,7 & 46,7 & 62,4 \\
registrado & 11,6 & 13,7 & 10,6 & 9,3 & 9,3 & 8,3 & 0,0 & 0,0 & 0,0 & 0,0 & 0,0 & 0,0 \\
$\begin{array}{l}\text { Patrón } \\
\text { Cuenta propia }\end{array}$ & 25,6 & 21,5 & 20,7 & 10,1 & 10,1 & 9,2 & 25,5 & 30,0 & 25,2 & 3,1 & 3,9 & 2,3 \\
$\begin{array}{l}\text { Trabajador familiar } \\
\text { sin remuneración }\end{array}$ & 0,4 & 0,0 & 0,0 & 0,2 & 0,2 & 0,0 & 1,2 & 1,5 & 0,5 & 3,7 & 3,4 & 1,5 \\
Total & 100 & 100 & 100 & 100 & 100 & 100 & 100 & 100 & 100 & 100 & 100 & 100 \\
\hline
\end{tabular}

Fuente: elaboración propia con base en EPH - INDEC (IV trimestres)

\section{Tamaño del establecimiento}

El tamaño del establecimiento indica cuál es la distribución de la fuerza de trabajo en función de los distintos establecimientos, clasificados según la cantidad de trabajadores que se desempeña en el mismo.

En promedio según los datos de la EPH (IV trimestres) entre 2004, 2008 y 2012, las grandes empresas -más de 40 ocupados- concentran algo menos que el $40 \%$ de la fuerza laboral, le siguen las pymes -de 6 a 40 ocupados- con 37,1\%, y luego los pequeños establecimientos y contrataciones particulares -1 a 5 ocupados- con poco más del $20 \%$. Cuando se analiza el tamaño del establecimiento en función del registro, se puede apreciar que en Argentina se perpetúa la lógica en la cual los establecimientos de menor cantidad de ocupados, más pequeños, concentran marcadamente el trabajo no registrado, (Tabla 8). 
Tabla 8.

Asalariados según condición de registro y tamaño del establecimiento. 18 a 59 años. 2004-2008$2012(\%)$

\begin{tabular}{lcccccc}
\hline & \multicolumn{3}{c}{ Asalariados registrados } & \multicolumn{4}{c}{ Asalariados no registrados } \\
\cline { 2 - 7 } & $\mathbf{2 0 0 4}$ & $\mathbf{2 0 0 8}$ & $\mathbf{2 0 1 2}$ & $\mathbf{2 0 0 4}$ & $\mathbf{2 0 0 8}$ & $\mathbf{2 0 1 2}$ \\
\hline Total & & & & & & \\
De 1 a 5 ocupados & 26,3 & 22,1 & 21,9 & 47,1 & 49,2 & 51,9 \\
De 6 a 40 ocupados & 38,6 & 37,1 & 35,5 & 40,2 & 38,8 & 35,7 \\
Más de 40 ocupados & 35,0 & 40,9 & 42,6 & 12,7 & 12,0 & 12,4 \\
Total & 100,0 & 100,0 & 100,0 & 100,0 & 100,0 & 100,0 \\
\hline
\end{tabular}

Fuente: elaboración propia con base en EPH - INDEC (IV trimestres)

Asimismo, la concentración de diversos niveles de calificación se verifica según los distintos tamaños de establecimientos. Mientras que en cantidad de trabajadores en los grandes establecimientos el orden es primero operativos, segundo técnicos, tercero profesionales y cuarto no calificados; para el caso de establecimientos de menos de 5 ocupados, también son mayoritariamente los trabajadores en puestos operativos, pero le siguen los no calificados, luego técnicos y finalmente profesionales. Esta tendencia se conserva al comparar varones y mujeres, con la excepción que proporcionalmente en pequeños establecimientos, la categoría de profesionales es más significativa para las mujeres (Tabla 9).

Tabla 9.

Nivel de calificación según tamaño del establecimiento. 18 a 59 años. 2004-2008-2012 (\%)

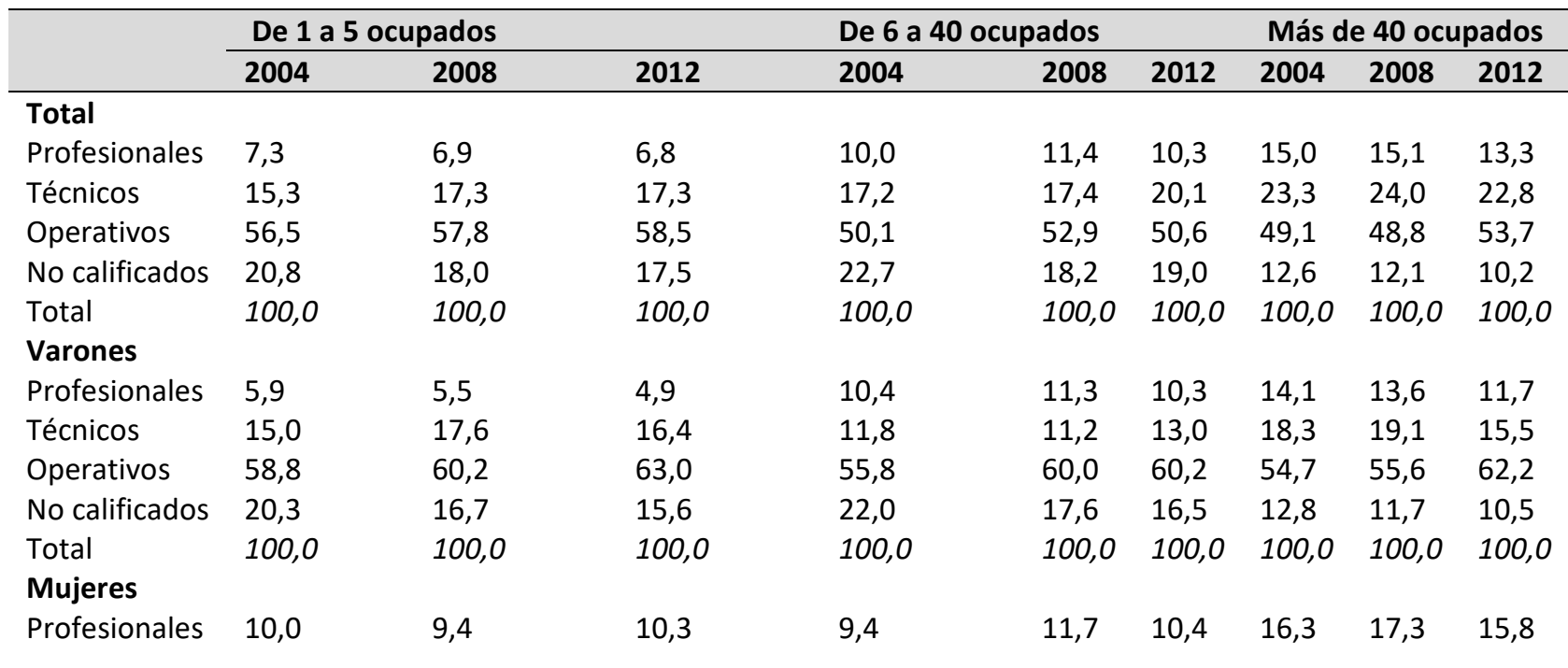




\begin{tabular}{|c|c|c|c|c|c|c|c|c|c|}
\hline & \multicolumn{2}{|c|}{ De 1 a 5 ocupados } & \multirow[b]{2}{*}{2012} & \multicolumn{3}{|c|}{ De 6 a 40 ocupados } & \multicolumn{3}{|c|}{ Más de 40 ocupados } \\
\hline & 2004 & 2008 & & 2004 & 2008 & 2012 & 2004 & 2008 & 2012 \\
\hline Técnicos & 15,7 & 16,8 & 18,9 & 24,7 & 26,6 & 29,5 & 30,8 & 31,4 & 34,0 \\
\hline Operativos & 52,4 & 53,4 & 49,5 & 42,2 & 42,5 & 37,7 & 40,6 & 38,7 & 40,6 \\
\hline No calificados & 21,8 & 20,4 & 21,2 & 23,7 & 19,2 & 22,3 & 12,3 & 12,7 & 9,6 \\
\hline Total & 100,0 & 100,0 & 100,0 & 100,0 & 100,0 & 100,0 & 100,0 & 100,0 & 100,0 \\
\hline
\end{tabular}

Fuente: elaboración propia con base en EPH - INDEC (IV trimestres)

\section{Rama de actividad}

En lo que sigue, se analiza la correlación entre el nivel educativo y la rama de actividad. Se busca dar cuenta de cuál es la composición de la fuerza de trabajo por ramas de actividad en función del máximo nivel educativo alcanzado por los trabajadores.

El total de ocupados que cuenta con el nivel educativo más bajo -hasta primario incompleto- se concentra en las ramas de construcción, comercio e industria para el caso de los varones y servicio doméstico y comercio en mujeres.

Cuando se analizan los datos para el resto de los trabajadores, se advierte que quienes poseen hasta secundario incompleto concentran su actividad en el comercio y la industria para la suma de ambos sexos, y si se disgrega entre varones y mujeres, industria y servicio doméstico respectivamente. En el tramo de hasta superior incompleto tanto varones como mujeres se concentran en el comercio y finalmente, la rama de actividad que presenta el mayor nivel educativo en ambos grupos es el sector público (ㅁabla 10).

Ahora bien, una de las relaciones más particulares, es la que se establece entre el nivel de calificación de la tarea y la rama de actividad. La composición de la estructura de calificaciones según las diversas ramas de actividad muestra que quienes carecen de calificación se agrupan en el caso de los varones en las ramas del comercio -40,5\%-, construcción -18,6\%- e industria $12,4 \%$ - y para las mujeres servicio doméstico $-54,4 \%$ - y comercio $-19,8 \%$-. Por su parte, los profesionales, tal como sucede según nivel educativo se concentran en la rama del sector público mayoritariamente (Tabla 11). 
Tabla 10.

Ocupados por rama de actividad según nivel educativo. 18 a 59 años. 2004-2008-2012 (\%)

\begin{tabular}{|c|c|c|c|c|c|c|c|c|c|c|c|c|}
\hline & \multicolumn{3}{|c|}{$\begin{array}{l}\text { Hasta primario } \\
\text { incompleto }\end{array}$} & \multicolumn{3}{|c|}{$\begin{array}{l}\text { Hasta secundario } \\
\text { incompleto }\end{array}$} & \multicolumn{3}{|c|}{$\begin{array}{l}\text { Hasta superior } \\
\text { incompleto }\end{array}$} & \multicolumn{3}{|c|}{ Superior completo } \\
\hline & 2004 & 2008 & 2012 & 2004 & 2008 & 2012 & 2004 & 2008 & 2012 & 2004 & 2008 & 2012 \\
\hline \multicolumn{13}{|l|}{ Total } \\
\hline Industria & 14,5 & 16,0 & 12,7 & 18,5 & 19,2 & 18,5 & 16,9 & 17,6 & 16,7 & 8,6 & 9,5 & 7,3 \\
\hline Construcción & 17,7 & 22,2 & 21,7 & 8,9 & 12,5 & 12,8 & 2,9 & 3,7 & 4,2 & 1,6 & 2,1 & 1,0 \\
\hline Servicio doméstico & 22,3 & 25,8 & 27,1 & 14,7 & 15,6 & 17,4 & 5,1 & 6,3 & 5,8 & 1,2 & 0,7 & 0,9 \\
\hline Comercio & 14,2 & 14,7 & 17,4 & 18,8 & 20,0 & 21,1 & 25,2 & 25,4 & 23,5 & 7,1 & 8,8 & 8,7 \\
\hline Transporte & 5,7 & 4,9 & 6,3 & 9,3 & 9,5 & 10,4 & 8,6 & 7,9 & 10,0 & 3,7 & 3,2 & 6,3 \\
\hline $\begin{array}{l}\text { Servicios perso- } \\
\text { nales y sociales }\end{array}$ & 8,3 & 8,4 & 4,8 & 9,1 & 7,1 & 6,2 & 12,2 & 10,9 & 9,9 & 22,9 & 22,1 & 16,6 \\
\hline Sector público & 15,2 & 5,7 & 5,9 & 15,7 & 10,2 & 8,4 & 17,5 & 16,4 & 18,0 & 42,5 & 41,1 & 46,9 \\
\hline Servicios modernos & 2,3 & 2,2 & 4,0 & 5,0 & 5,9 & 5,2 & 11,7 & 11,8 & 11,9 & 12,3 & 12,5 & 12,3 \\
\hline Total & 100 & 100 & 100 & 100 & 100 & 100 & 100 & 100 & 100 & 100 & 100 & 100 \\
\hline \multicolumn{13}{|l|}{ Varones } \\
\hline Industria & 20,3 & 22,9 & 17,0 & 23,7 & 24,0 & 23,9 & 22,0 & 23,2 & 20,7 & 13,9 & 15,0 & 13,1 \\
\hline Construcción & 31,4 & 36,7 & 35,6 & 14,1 & 19,1 & 19,4 & 4,9 & 6,3 & 6,9 & 3,5 & 4,5 & 2,0 \\
\hline Servicio doméstico & 0,5 & 0,3 & 0,0 & 0,5 & 0,2 & 0,6 & 0,3 & 0,1 & 0,2 & 0,1 & 0,0 & 0,0 \\
\hline Comercio & 17,2 & 18,0 & 24,0 & 22,5 & 21,7 & 23,2 & 24,9 & 24,9 & 23,5 & 8,5 & 12,0 & 10,6 \\
\hline Transporte & 10,1 & 8,1 & 10,2 & 14,3 & 13,9 & 14,8 & 11,6 & 11,4 & 14,3 & 5,6 & 5,7 & 9,5 \\
\hline $\begin{array}{l}\text { Servicios perso- } \\
\text { nales y sociales }\end{array}$ & 6,9 & 7,0 & 4,9 & 7,1 & 6,2 & 4,8 & 8,8 & 8,0 & 6,2 & 1 & 13,3 & 9,5 \\
\hline Sector público & 11,5 & 5,3 & 5,0 & 11,7 & 9,3 & 7,6 & 15,5 & 14,9 & 16,5 & 36,7 & 34,5 & 37,7 \\
\hline Servicios modernos & 2,1 & 1,7 & 3,2 & 6,1 & 5,7 & 5,8 & 12,0 & 11,2 & 11,8 & 14,5 & 15,0 & 17,6 \\
\hline Total & 100 & 100 & 100 & 100 & 100 & 100 & 100 & 100 & 100 & 100 & 100 & 100 \\
\hline \multicolumn{13}{|l|}{ Mujeres } \\
\hline Industria & 7,1 & 5,7 & 6,2 & 9,9 & 10,4 & 8,3 & 10,4 & 10,3 & 11,1 & 5,2 & 6,1 & 3,7 \\
\hline Construcción & 0,1 & 0,3 & 0,6 & 0,1 & 0,5 & 0,4 & 0,4 & 0,5 & 0,4 & 0,4 & 0,7 & 0,4 \\
\hline Servicio doméstico & 50,2 & 64,1 & 68,5 & 38,3 & 43,9 & 48,9 & 11,2 & 14,3 & 13,6 & 1,9 & 1,1 & 1,4 \\
\hline Comercio & 10,3 & 9,8 & 7,3 & 12,6 & 16,9 & 17,2 & 25,6 & 26,0 & 23,6 & 6,2 & 6,8 & 7,5 \\
\hline Transporte & 0,0 & 0,3 & 0,2 & 1,1 & 1,4 & 2,0 & 4,7 & 3,6 & 4,0 & 2,5 & 1,6 & 4,3 \\
\hline $\begin{array}{l}\text { Servicios perso- } \\
\text { nales y sociales }\end{array}$ & 10,0 & 10,5 & 4,7 & 12,4 & 8,9 & 9,0 & 16,5 & 14,5 & 15,1 & 26,7 & 27,5 & 20,9 \\
\hline Sector público & 19,9 & 6,4 & 7,3 & 22,5 & 11,8 & 10,1 & 20,0 & 18,2 & 20,1 & 46,2 & 45,2 & 52,7 \\
\hline Servicios modernos & 2,4 & 3,0 & 5,2 & 3,2 & 6,2 & 4,1 & 11,2 & 12,6 & 12,1 & 10,9 & 11,0 & 8,9 \\
\hline Total & 100 & 100 & 100 & 100 & 100 & 100 & 100 & 100 & 100 & 100 & 100 & 100 \\
\hline
\end{tabular}

Fuente: elaboración propia con base en EPH - INDEC (IV trimestres) 
Tabla 11.

Rama de actividad asalariados registrados según nivel de calificación. 18 a 59 años. 2004-2008$2012(\%)$

\begin{tabular}{|c|c|c|c|c|c|c|c|c|c|c|c|c|}
\hline & \multicolumn{3}{|c|}{ Profesionales } & \multicolumn{3}{|c|}{ Técnicos } & \multicolumn{3}{|c|}{ Operativos } & \multicolumn{3}{|c|}{ No calificados } \\
\hline & 2004 & 2008 & 2012 & 2004 & 2008 & 2012 & 2004 & 2008 & 2012 & 2004 & 2008 & 2012 \\
\hline \multicolumn{13}{|l|}{ Total } \\
\hline Industria & 6,8 & 9,8 & 6,1 & 11,1 & 9,6 & 6,6 & 23,0 & 24,2 & 23,3 & 8,9 & 8,0 & 7,4 \\
\hline Construcción & 2,2 & 2,6 & 2,3 & 1,6 & 2,9 & 0,6 & 6,3 & 9,5 & 9,4 & 8,4 & 7,2 & 7,0 \\
\hline Servicio doméstico & 0,0 & 0,0 & 0,0 & 0,1 & 0,2 & 0,3 & 0,2 & 0,2 & 0,0 & 30,8 & 34,1 & 34,5 \\
\hline Comercio & 5,0 & 6,1 & 4,8 & 6,9 & 6,3 & 6,7 & 20,3 & 21,6 & 20,0 & 26,3 & 28,3 & 29,6 \\
\hline Transporte & 3,6 & 3,1 & 10,3 & 3,2 & 5,4 & 7,8 & 13,0 & 10,6 & 12,4 & 3,0 & 3,7 & 3,5 \\
\hline $\begin{array}{l}\text { Servicios perso- } \\
\text { nales y sociales }\end{array}$ & 19,5 & 17,4 & 13,8 & 26,5 & 26,4 & 21,8 & 10,1 & 8,5 & 7,1 & 7,8 & 7,3 & 7,1 \\
\hline Sector público & 45,8 & 41,6 & 45,1 & 39,8 & 38,2 & 43,4 & 17,7 & 15,7 & 17,8 & 10,6 & 6,3 & 6,3 \\
\hline Servicios modernos & 17,0 & 19,4 & 17,4 & 10,9 & 11,0 & 12,8 & 9,4 & 9,7 & 9,9 & 4,2 & 5,1 & 4,6 \\
\hline Total & 100 & 100 & 100 & 100 & 100 & 100 & 100 & 100 & 100 & 100 & 100 & 100 \\
\hline \multicolumn{13}{|l|}{ Varones } \\
\hline Industria & 9,8 & 14,9 & 9,4 & 18,6 & 15,5 & 12,1 & 26,4 & 27,4 & 26,6 & 14,1 & 13,6 & 9,4 \\
\hline Construcción & 3,5 & 4,7 & 3,7 & 3,1 & 5,7 & 1,1 & 9,3 & 13,5 & 13,0 & 19,6 & 18,2 & 17,9 \\
\hline Servicio doméstico & 0,0 & 0,0 & 0,0 & 0,0 & 0,0 & 0,0 & 0,2 & 0,1 & 0,0 & 1,0 & 0,3 & 1,6 \\
\hline Comercio & 6,6 & 7,7 & 7,2 & 11,3 & 9,9 & 12,0 & 19,3 & 20,1 & 18,2 & 37,2 & 39,6 & 44,7 \\
\hline Transporte & 3,9 & 4,8 & 14,4 & 5,7 & 11,3 & 13,1 & 16,9 & 13,0 & 15,5 & 5,8 & 8,8 & 6,9 \\
\hline $\begin{array}{l}\text { Servicios perso- } \\
\text { nales y sociales }\end{array}$ & 16,3 & 10,0 & 10,0 & 18,5 & 17,7 & 11,5 & 6,7 & 5,6 & 4,8 & 7,3 & 7,8 & 5,7 \\
\hline Sector público & 42,1 & 35,1 & 36,0 & 29,6 & 25,3 & 31,3 & 12,5 & 12,7 & 13,3 & 9,9 & 7,1 & 7,6 \\
\hline Servicios modernos & 17,8 & 22,7 & 19,3 & 13,2 & 14,6 & 18,8 & 8,7 & 7,5 & 8,6 & 5,2 & 4,6 & 6,1 \\
\hline Total & 100 & 100 & 100 & 100 & 100 & 100 & 100 & 100 & 100 & 100 & 100 & 100 \\
\hline \multicolumn{13}{|l|}{ Mujeres } \\
\hline Industria & 3,0 & 4,3 & 2,5 & 4,9 & 4,7 & 2,9 & 16,1 & 17,3 & 15,1 & 5,1 & 4,4 & 6,1 \\
\hline Construcción & 0,7 & 0,3 & 0,9 & 0,4 & 0,7 & 0,3 & 0,3 & 0,9 & 0,5 & 0,2 & 0,1 & 0,3 \\
\hline Servicio doméstico & 0,0 & 0,0 & 0,0 & 0,1 & 0,3 & 0,4 & 0,3 & 0,3 & 0,0 & 52,9 & 55,8 & 54,6 \\
\hline Comercio & 3,1 & 4,3 & 2,3 & 3,2 & 3,4 & 3,2 & 22,3 & 24,7 & 24,7 & 18,2 & 21,0 & 20,3 \\
\hline Transporte & 3,3 & 1,2 & 5,9 & 1,1 & 0,6 & 4,2 & 5,2 & 5,5 & 4,7 & 1,0 & 0,4 & 1,5 \\
\hline $\begin{array}{l}\text { Servicios perso- } \\
\text { nales y sociales }\end{array}$ & 23,5 & 25,4 & 17,9 & 33,1 & 33,5 & 28,8 & 17,0 & 14,8 & 12,8 & 8,2 & 7,0 & 7,9 \\
\hline Sector público & 50,4 & 48,5 & 54,9 & 48,3 & 48,7 & 51,6 & 28,1 & 22,1 & 29,1 & 11,1 & 5,9 & 5,5 \\
\hline Servicios modernos & 16,0 & 15,9 & 15,5 & 9,0 & 8,1 & 8,7 & 10,7 & 14,4 & 13,1 & 3,4 & 5,5 & 3,8 \\
\hline Total & 100 & 100 & 100 & 100 & 100 & 100 & 100 & 100 & 100 & 100 & 100 & 100 \\
\hline
\end{tabular}

Fuente: elaboración propia con base en EPH - INDEC (IV trimestres)

\section{Conclusiones}

Cuando se analiza la evolución de algunos de los principales indicadores económicos en el período 2004-2012, si bien se evidencia cierta mejoría en relación a la formalización del empleo y los niveles de ocupación en general, marcados rasgos de un mercado laboral segmentado se 18 
siguen haciendo visibles. Ello, pone de manifiesto que el crecimiento económico no sería la única herramienta que permite combatir la precariedad.

Subsisten características que evidencian un mercado laboral segmentado como la existencia de trabajadores cuentapropistas y asalariados no registrados que se concentran en los segmentos de menor nivel educativo, ocupados preponderantemente en establecimientos pequeños. De modo que, quienes sufren más la precariedad laboral son aquellos que debido a su menor nivel educativo carecen de la posibilidad de inserción en otros puestos. Ello, a causa de un mercado de trabajo que ofrece escasos e insuficientes puestos laborales de los cuales una parte importante revisten la categoría de precarios. Por consiguiente, es válido pensar que la precariedad es del puesto de trabajo y no del trabajador, a partir de lo cual, las condiciones de informalidad/precariedad no son atribuibles a las "condiciones de empleabilidad" de los sujetos individuales, sino que se fundan en la estructura económica informal y segmentada.

La segmentación también se hace visible en términos de género. Las mujeres participan notoriamente menos en el mercado laboral que los varones, tendencia que se mantiene en el período 2004-2012. Ellas, asimismo, sufren en forma más marcada la desocupación y esta mayor condena recae, mayoritariamente, en aquellas de menor nivel educativo.

Finalmente, resulta valioso mencionar que todavía, los trabajadores de calificación profesional revisten un registro que duplica el nivel de la de aquellos no calificados, lo cual perpetúa una lógica de mercado dual en la que conviven segmentos más y menos favorecidos.

\section{Referencias}

Arceo, N., González, M., Mendizábal, N., y Basualdo E. (2010). La economía argentina de la posconvertibilidad en tiempos de crisis mundial. Recuperado de https://www.econbiz.de/Record/la-econom\%C3\%ADa-argentina-de-laposconvertibilidad-en-tiempos-de-crisis-mundial-arceonicol\%C3\%A1s/10009356424

Azpiazu, D. y Schorr, M. (2010). Hecho en Argentina. Industria y economía, 1976-2007. Recuperado de http://www.sigloxxieditores.com.ar/fichaLibro.php?libro=978-987$\underline{629-124-8}$

Bennholdt-Thomsen, V., y Garrido, A. (1981).Marginalidad en América Latina. Una crítica de la teoría. Revista Mexicana de Sociología, 43(4), 1505-1546. Recuperado de https://doi.org/10.2307/3540058 
Bertranou, F., Casanova, L., Jiménez, M., y Jiménez, M. (2013). Informalidad, calidad del empleo y segmentación laboral en Argentina (Documentos de trabajo, № 2). Recuperado del sitio de internet de la Organización Internacional de Trabajo: http://www.ilo.org/wcmsp5/groups/public/---americas/---ro-lima/---ilobuenos aires/documents/publication/wcms 228741.pdf

Calero, A., y Sorokin, I. (2013). Condiciones de trabajo y calidad del empleo en la provincia de Buenos Aires en el período 2003-2011: Análisis de los determinantes de los puestos de trabajo con bajas remuneraciones en Ministerio de Trabajo de la provincia de Buenos Aires. En Maestre, A., y Chicatún, L. (Comps). Tercer concurso Bialet Massé (pp.4-5). Recuperado de http://www.trabajo.gba.gov.ar/informacion/masse/Resumen Tercer CONCURSO.p $\underline{\mathrm{df}}$

Castel, R. (1997). Las metamorfosis de la cuestión social. Una crónica del salariado. Recuperado de $\quad$ https://www.amazon.com/Las-Metamorfosis-Cuestion-SocialIntegration/dp/9501254577

Castel, R. (2010). El ascenso de las incertidumbres. Trabajo, protecciones, estatuto del individuo. Recuperado de http://www.fce.com.ar/archivos/pdfs/Castel.pdf

Fernández-Huerga, E. (2010). La teoría de la segmentación del mercado de trabajo: enfoques, situación actual y perspectivas de futuro. Investigación Económica, LXIX(273), 115150. Recuperado de http://www.redalyc.org/pdf/601/60114744004.pdf

Groisman, F. (2010). La persistencia de la segregación residencial socioeconómica en Argentina. Estudios demográficos y urbanos, 25(2). Recuperado de http://www.redalyc.org/articulo.oa?id=31221521005

Groisman, F. (2013). Gran Buenos Aires: Polarización de ingresos, clase media e informalidad laboral, 1974-2010. Revista CEPAL, 109. Recuperado de http://www.cepal.org/publicaciones/xml/1/49521/RVE109Groiman.pdf

Groisman, F., Beccaria L. y Monsalvo P. (2006). Segmentación del mercado de trabajo y pobreza en Argentina. Recuperado de http://www.ungs.edu.ar/ms ici/wpcontent/uploads/2015/10/Beccaria Groisman Monsalvo.pdf

Groisman, F., y Beccaria, L. (2009). Argentina Desigual. Recuperado de http://www.ungs.edu.ar/areas/publicaciones/208/argentina-desigual.html

20

María Eugenia Sconfienza

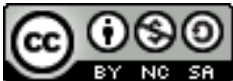

Revista Economía y Sociedad by Universidad Nacional is licensed under a CreativeCommons Reconocimiento-NoComercialCompartirlgual 4.0 Internacional License.

Creado a partir de la obra en http://www.revistas.una.ac.cr/index.php/economia 
Häusermann, S. y Schwander, H. (April 2010). Explaining welfare preferences in dualized societies. Paper preparado para el panel The Dualization of European Societies?, 17th Conference of Europeanists, Montréal. Recuperado de http://www.schwander-hanna.ch/files/Hausermann Schwander CES2010.pdf

Marshall A. y Perelman L. (2013). El empleo industrial: balance de una década 2003-2012 (Serie Documentos para discusión del PESEI $N^{\circ}$ 9). Recuperado del sitio web Instituto de Desarrollo Económico y Social: http://ides.org.ar/archivos/6322

Nun, J. (1969). Superpoblación relativa, ejército industrial de reserva y masa marginal. Revista Latinoamericana de Sociología, 5(2). Recuperado de http://repositorio.cepal.org/bitstream/handle/11362/7934/S7100908 es.pdf?sequ ence $=1 \&$ isAllowed $=y$

Piore, M. J. (1983). Notas para una teoría de la estratificación del mercado de trabajo en L. Toharia, El mercado de trabajo: Teorías y aplicaciones. Recuperado de https://www.amazon.es/El-mercado-trabajo-Teor\%C3\%ADasaplicaciones/dp/BOOIAKNJPK

Sconfienza, M. E. (2016). Demanda laboral y segmentos vulnerables. En Serie Empleo, desempleo \& políticas de empleo $N^{\circ} 25$. Recuperado de http://www.ceilconicet.gov.ar/wp-content/uploads/2016/08/EDPE25-Sconfienza.pdf

Zuazúa, N. (2006). La estrategia de la administración Kirchner para enfrentar los problemas del mercado de empleo (Análisis de Coyuntura $\mathrm{N}^{\circ} 12$ ). Recuperado del sitio de internet del Centro Interdisciplinario para el estudio de políticas públicas: http://www.ciepp.org.ar/index.php?page=shop.getfile\&file id=116\&product id=11 6\&option=com virtuemart\&ltemid=2\&lang=es 